\title{
Experimental observation of the Bogoliubov transformation for a Bose-Einstein condensed gas
}

\author{
J.M. Vogels, K. Xu, C. Raman,7 J. R. Abo-Shaeer, and W. Ketterlef \\ Department of Physics, MIT-Harvard Center for Ultracold Atoms, \\ and Research Laboratory of Electronics, Massachusetts Institute of Technology, Cambridge, MA 02139
}

\begin{abstract}
Phonons with wavevector $q / \hbar$ were optically imprinted into a Bose-Einstein condensate. Their momentum distribution was analyzed using Bragg spectroscopy with a high momentum transfer. The wavefunction of the phonons was shown to be a superposition of $+q$ and $-q$ free particle momentum states, in agreement with the Bogoliubov quasiparticle picture.
\end{abstract}

PACS numbers: 05.30.Jp, 03.75.Fi, 32.80.-t, 67.40.Db

The pioneering work of Bogoliubov in 1947 constitutes the first microscopic theory that attributes superfluidity to Bose-Einstein condensation [1]. As described by Einstein noninteracting bosons condense [2], but they are not superfluid. However, Bogoliubov showed that with weak interactions the condensate will exhibit superfluidity. Repulsive interactions change the elementary excitations at long wavelengths from free particles into phonons which, according to the Landau criterion, leads to superfluidity [3]. The main step in the non-perturbative treatment is the Bogoliubov transformation

$$
\begin{aligned}
& b_{+q}^{\dagger}:=u_{q} a_{+q}^{\dagger}+v_{q} a_{-q} \\
& b_{+q}:=u_{q} a_{+q}+v_{q} a_{-q}^{\dagger}
\end{aligned}
$$

which expresses the creation and annihilation operators $b_{q}^{\dagger}$ and $b_{q}$ for Bogoliubov quasiparticles in terms of creation and annihilation operators $a_{q}^{\dagger}$ and $a_{q}$ for free particles in momentum states $q$. This transformation also plays a crucial role in general relativity, where it connects

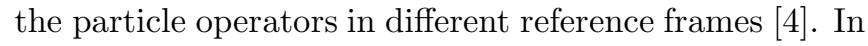
this paper we experimentally verify the Bogoliubov transformation by generating quasiparticles with momentum $+q$ and observing that they are a superposition of $+q$ and $-q$ momentum states of free particles.

Following a recent suggestion of Brunello et al. [5], we perform an experiment that combines the two regimes of Bragg spectroscopy, where the momentum imparted to the atoms is either smaller [6] or larger [7] than the sound velocity $c$ times the mass $m$ of the atoms. An optical lattice moving through the condensate at the sound velocity imprinted phonons with wavelengths equal to the spatial period of the lattice [6]. This was accomplished by intersecting two laser beams at a small angle and choosing their frequency difference to be equal to the phonon frequency. The momentum analysis of the phonon wavefunction was performed by a second Bragg pulse consisting of counterpropagating laser beams that

\footnotetext{
${ }^{*}$ Current Address: School of Physics Georgia Institute of Technology, Atlanta, GA 30332

${ }^{\dagger}$ Group website: http://cua.mit.edu/ketterle_group/
}

transferred a large momentum $Q$ (two photon recoils) to atoms with initial momentum $p$. In the limit $Q \gg p$, $m c$, the resonance frequency $\nu$ is equal to the kinetic energy transferred: $h \nu=Q^{2} / 2 m+Q p / m$, where the second term is simply the Doppler shift 4 , 8, 9. The resulting frequency spectrum shows three peaks corresponding to the three momentum components $(0,+q$ and $-q)$ of a condensate with phonons (see Fig. 2) (5].

The Bogoliubov spectrum for the energy of elementary excitations is 10

$$
\varepsilon(q)=\sqrt{q^{2} c^{2}+\left(\frac{q^{2}}{2 m}\right)^{2}}
$$

where $c=\sqrt{4 \pi \hbar^{2} a n} / m, a$ is the scattering length, and $n$ is the density. The amplitudes $u_{q}$ and $v_{q}$ in Eq. 1 are given by

$$
u_{q}, v_{q}= \pm \frac{\varepsilon(q) \pm \frac{q^{2}}{2 m}}{2 \sqrt{\varepsilon(q) \frac{q^{2}}{2 m}}}
$$

The non-trivial aspect of the Bogoliubov transformation manifests itself only at low momenta $(q \ll m c)$, where both amplitudes $u_{q}$ and $v_{q}$ are significant. In this regime, the excitations are characterized by $u \sim$ $-v \sim \sqrt{m c / 2 q} \gg 1$ and $\varepsilon(q) \sim q c$. Such excitations are phonons, each involving many particles moving in both directions. At high momenta $(q \gg m c), u_{q} \sim 1$, $v_{q} \sim 0$ and $\varepsilon(q)=q^{2} / 2 m+m c^{2}$. These excitations are free particles with an energy shift equal to the chemical potential $\mu=m c^{2}$.

The phonon creation operator $b_{+q}^{\dagger}$ (Eq. 1) is a superposition of the creation operator $a_{+q}^{\dagger}$ for particles moving in the $+q$ direction and the annihilation operator $a_{-q}$ for particles moving in the $-q$ direction, yet the creation of a phonon in the condensate implies an increase of particles moving in both the $+q$ and $-q$ direction. Indeed, simple operator algebra shows that a condensate with $l$ excitations $b_{+q}^{\dagger l} / \sqrt{l !}\left|\Psi_{0}\right\rangle$ contains $l u_{q}^{2}+v_{q}^{2}$ free particles moving with momentum $+q$ and $(l+1) v_{q}^{2}$ free particles with momentum $-q$. In its ground state the condensate contains $v_{q}^{2}$ pairs of atoms with momenta $+q$ and 
$-q$. These pairs constitute the quantum depletion in the condensate wavefunction [10]

$$
\left|\Psi_{0}\right\rangle=\prod_{q \neq 0} \frac{1}{u_{q}} \sum_{j=0}^{\infty}\left(-\frac{v_{q}}{u_{q}}\right)^{j}\left|n_{-q}=j, n_{+q}=j\right\rangle .
$$

where the remaining atoms are in the $q=0$ momentum state. When $a_{+q}^{\dagger}$ and $a_{-q}$ act on $\Psi_{0}$, terms with large occupation numbers $j$ are enhanced: $a_{+q}^{\dagger}\left|n_{-q}=j, n_{+q}=j\right\rangle=\sqrt{j+1}\left|n_{-q}=j, n_{+q}=j+1\right\rangle$, $a_{-q}\left|n_{-q}=j, n_{+q}=j\right\rangle=\sqrt{j}\left|n_{-q}=j-1, n_{+q}=j\right\rangle$. In addition, $-q$ and $+q$ atoms appear only in pairs. Together, these two effects cause both $a_{+q}^{\dagger}$ and $a_{-q}$ to increase the number of atoms in both the $-q$ and $+q$ states.

The experiments were performed with condensates of $3 \times 10^{7}$ sodium atoms in a magnetic trap with radial and axial trapping frequencies of $37 \mathrm{~Hz}$ and $7 \mathrm{~Hz}$, respectively [11]. The condensate had a peak density of $1.0 \times 10^{14} \mathrm{~cm}^{-3}$ corresponding to a chemical potential of $\mu=h \cdot 1.5 \mathrm{kHz}$, a sound velocity of $c=5 \mathrm{~mm} / \mathrm{s}$, and a Thomas-Fermi radial radius of $32 \mu \mathrm{m}$. The Bragg beams for the optical lattices were generated from a common source of laser light $1.7 \mathrm{GHz}$ red-detuned from the $3 S_{1 / 2}$ $|F=1\rangle$ to $3 P_{3 / 2}\left|F^{\prime}=0,1,2\right\rangle$ transitions. The lattices were moved radially through the cloud. This was done to avoid the high collisional density along the axial direction, where outcoupled atoms would have undergone elastic collisions with the condensate [12]. The Bragg beams for imprinting the phonons were at an angle of 32 mrad with respect to one another, resulting in a lattice spacing of $\sim 9 \mu \mathrm{m}$ and a recoil momentum of $q=m \cdot 1.9$ $\mathrm{mm} / \mathrm{s}$. The beams propagated at an angle of $0.4 \mathrm{rad}$ with respect to the longitudinal axis of the condensate and were linearly polarized perpendicular to it. The frequency difference ("excitation frequency") between the two beams was chosen to be $400 \mathrm{~Hz}$, corresponding to the frequency of the phonons. The excitation pulse had to be long enough to ensure sufficient frequency resolution in order to selectively excite $+q$ phonons, and no $-q$ phonons (see below). However, the excitation pulse also had to be shorter than the transit time of phonons through the condensate, since phonons accelerate when they move through regions of varying density. We chose small-angle Bragg beams $3 \mathrm{~ms}$ in duration with intensity $0.05 \mathrm{~mW} / \mathrm{cm}^{2}$, corresponding to a two-photon Rabi frequency of $50 \mathrm{~Hz}$.

The momentum analysis of the phonons was performed with two counterpropagating beams that imparted a recoil momentum of $Q=m .59 \mathrm{~mm} / \mathrm{s}$ onto the outcoupled atoms. The beams were polarized parallel to the longitudinal axis of the condensate to suppress superradiant emission 13. A frequency difference ("probe frequency") of $100 \mathrm{kHz}$ between the two beams corresponded to the kinetic energy needed for atoms initially at rest to reach this recoil momentum. To gain recoil momentum $+Q$, atoms with initial momentum $+q$ were resonant at 107 $\mathrm{kHz}$ (Fig. 1 1a). Atoms with momentum $-q$ were resonant at $94 \mathrm{kHz}$ (Fig. 1 b). In our experiment, a retroreflected

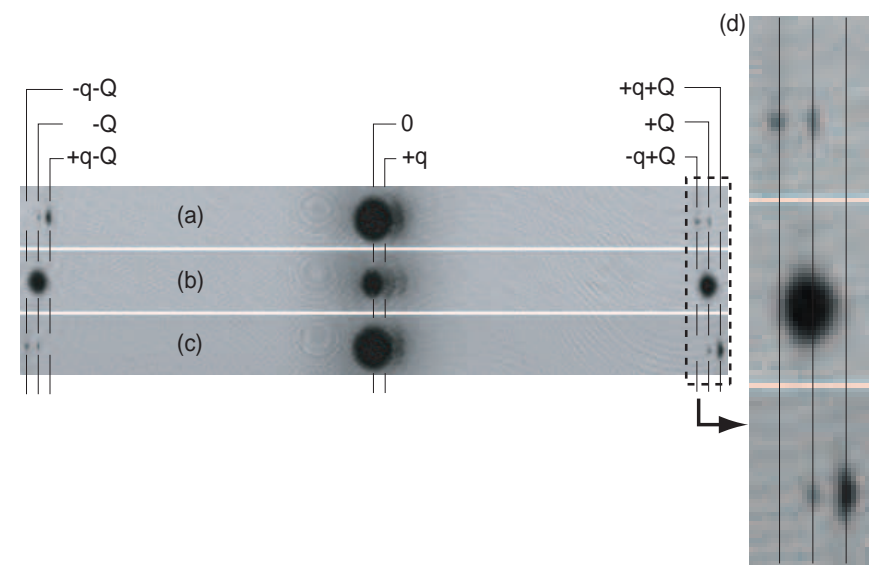

FIG. 1: Momentum distribution of a condensate with phonons. After imprinting $+q$ phonons into the condensate, momentum analysis via Bragg spectroscopy transfers a momentum $\pm Q$ (two photon recoil) to the atoms. Absorption images after $40 \mathrm{~ms}$ time of flight in (a), (b), and (c) show the condensate in the center and outcoupled atoms to the right and left for probe frequencies of 94, 100, and $107 \mathrm{kHz}$, respectively. The small clouds centered at $+q$ are phonons that were converted to free particles. The size of the images is $25 \times 2.2$ mm. (d) The outlined region in (a) - (c) is magnified, and clearly shows outcoupled atoms with momenta $Q \pm q$, implying that phonons with wavevector $q / \hbar$ have both $+q$ and $-q$ free particle momentum components.

beam containing both optical frequencies resulted in two optical lattices moving at the same speed in opposite directions. This led to simultaneous outcoupling of $+q$ and $-q$ atoms in opposite directions. These large-angle Bragg beams were pulsed on for $0.5 \mathrm{~ms}$. The probe pulse had to be long enough to selectively excite atoms with $\pm q$ momentum, but also shorter than $h / \mu$ for the mean field energy to be negligible during the readout. Each frequency component had an intensity of $0.7 \mathrm{~mW} / \mathrm{cm}^{2}$, corresponding to a two-photon Rabi frequency of $700 \mathrm{~Hz}$. Subsequently, the trap was turned off and a resonant absorption image was taken after $40 \mathrm{~ms}$ of ballistic expansion.

Fig. 1 shows typical absorption images for various probe frequencies. The quasiparticle nature of the phonons was directly evident (see outlined region) in the time-of-flight distribution through the presence of the peaks at momenta $\pm q+Q$. These peaks had well defined momentum because the outcoupled atoms left the condensate quickly (during this time the atoms' velocity changed by less than the speed of sound 14 ). This "photograph" of the Bogoliubov transformation is the central result of this paper.

We now discuss the different momentum components distinguishable in Fig. 1:

(1) The original condensate is in the center.

(2) The condensate is asymmetrically extended towards positive momenta. This is due to imprinted phonons of momentum $q$ that were converted to free par- 


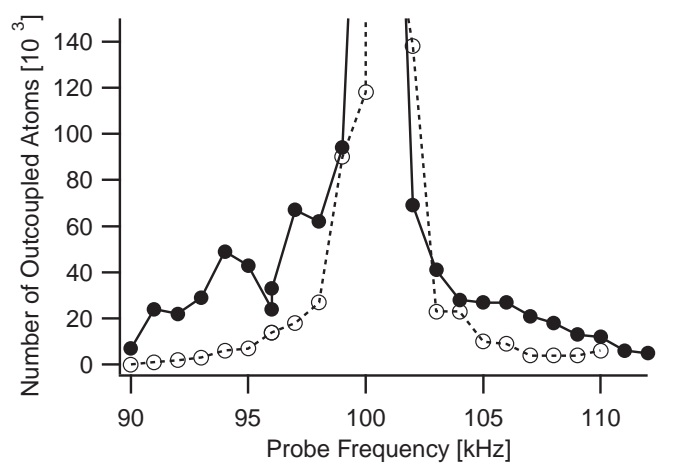

FIG. 2: Bragg spectrum of a condensate with $(\bullet)$ and without (o) phonon excitation. The number of outcoupled atoms vs. the probe frequency is shown. The excitation of phonons creates sidebands on both sides of the central condensate peak. Around $100 \mathrm{kHz}$, the absorption images were saturated due to high optical density.

ticles during the ballistic expansion. Previously this signature was used to determine the structure factor of a condensate [6]. There is no momentum component in the opposite direction, because we did not create $-q$ phonons (see below). The smearing of the observed momentum distribution may be caused by acceleration due to the inhomogeneous density distribution of the condensate when the trap was switched off.

(3) The two components with momentum $+Q$ and $-Q$ are atoms outcoupled from the condensate at rest. The symmetry of the $\pm Q$ peaks, as well as the position of the main condensate, served as an indicator as to whether the condensate was undergoing dipole oscillation during the experiment. Images that showed such "sloshing" (caused by technical noise) were excluded from further analysis.

(4) Atoms at $+q+Q$ (Fig. 1c) and $+q-Q$ (Fig. 1a) are coupled out from the $+q$ component of the phonons.

(5) Atoms at $-q-Q$ (Fig. 1c) and $-q+Q$ (Fig. 1a) are coupled out from the $-q$ component of the phonons.

Quantitative information was obtained by scanning the probe frequency and measuring the number of outcoupled atoms in the three peaks around $-Q$ (See Fig. 1). Without phonons only the condensate peak is observed. The excitation of phonons at wavevector $+q$ creates momentum sidebands at momentum $\pm q$. The $-q$ peak is expected to be smaller by a factor $v_{q}^{2} / u_{q}^{2}$. This effect is evident in Fig. 2, but with a poor signal-to-noise ratio.

The excitation of the phonons was characterized by scanning the frequency difference of the small angle Bragg beams (Fig. 3a) and measuring the number of outcoupled atoms at $+q-Q$. The probe frequency was kept at $94 \mathrm{kHz}$ because the momenta of the excited phonons remained fixed. The observation of two distinct peaks (corresponding to $+q$ at $400 \mathrm{~Hz}$ and $-q$ phonons at $400 \mathrm{~Hz}$, respectively) confirms that there was sufficient resolution to excite only $+q$ phonons and suppress the off-resonant excitation of $-q$ phonons. The higher peak (a)

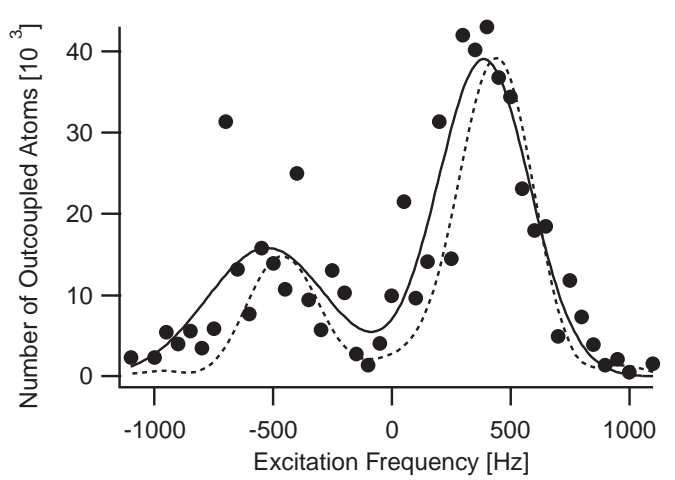

(b)

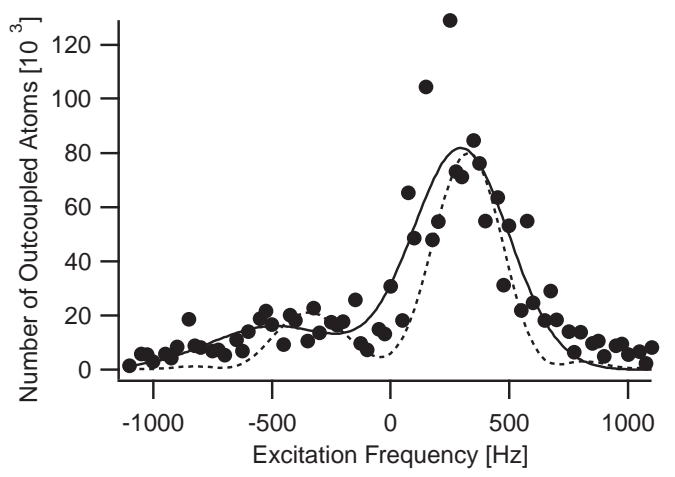

FIG. 3: Phonon excitation spectrum. Atoms with initial momentum $+q$ were detected by setting the probe frequency to $94 \mathrm{kHz}$ and measuring the number of atoms with momentum $-Q+q$. The two peaks reflect that phonons with $+q / \hbar$ and $-q / \hbar$ wavevectors have free particle components with momentum $+q$. Spectra were taken at: (a) high density $\left(1.0 \times 10^{14}\right.$ $\left.\mathrm{cm}^{-3}\right)$ and (b) low density $\left(0.5 \times 10^{14} \mathrm{~cm}^{-3}\right)$. The solid line, a fit to the sum of two Gaussians, is intended to guide the eye. The dashed line is the theoretical prediction.

represents the $u$-component of $+q$ phonons excited at positive excitation frequencies. When the excitation frequency became negative, $-q$ phonons were excited. This second peak represents the $v$-component of the phonons.

According to Eq. 3 the ratio of the two peaks, $v_{q}^{2} / u_{q}^{2}$, should be smaller at lower density. This is confirmed in Fig. 3b, which shows the excitation spectrum at low density. The density was lowered by a factor of 2 by weakening the axial trap frequency to $4 \mathrm{~Hz}$ and reducing the number of atoms in the condensate by a factor of 3 . The scatter in the data could be due to residual sloshing, shot-to-shot fluctuations in the size of the condensate, or non-linear effects (see below).

The dashed line is a the theoretical prediction with no free parameters (except the vertical scale). It was obtained by integrating Eq. 3 over the inhomogeneous density distribution of the condensate and by accounting for the finite length of the square excitation pulse, which broadens the line and causes the extra sidelobes around $900 \mathrm{~Hz}$. The theory assumes the validity of a perturbative approach, i.e. that the excitation pulse is weak.

Ideally, both Bragg pulses should affect only a few per- 
cent of the atoms, as in previous experiments $[6,7$. However, because our signal was the product of the two outcoupling efficiencies, we needed to work at much higher outcoupled fractions. We estimate that the excitation pulse transferred 10 of the condensate atoms into the phonon state, and that the probe pulse outcoupled $40 \%$ of these atoms on resonance.

Both Bragg processes (stimulated Rayleigh scattering) should depend only on the product of the two intensities. Therefore, changing the sign of the excitation frequency should not affect the number of phonons generated (assuming a stationary condensate). However, when the sign of the excitation frequency was changed in our experiment, the observed number of phonons differed. This is most likely due to superradiance 13 , which is sensitive to the individual intensities. Therefore, the asymmetry in phonon number was eliminated by ensuring equal intensities in the two beams. Additionally, there was a substantial loss $(\sim 50 \%)$ of condensate atoms due to superradiant Rayleigh scattering. Both superradiant effects could be further suppressed using light further detuned from resonance. However, in our case this would have required an additional laser. Given these experimental limitations, the agreement between experiment and theory in Fig. 3 is satisfactory.

In this work we have used large-angle Bragg pulses to analyze the momentum structure of the phonon wave- function. In principle, this could have also been achieved by removing the mean-field interaction within a time $h / \varepsilon(q)$ and then probing the velocity distribution of the particles. This is not possible with ordinary ballistic expansion because the reduction of the interactions is too slow, taking place on a time scale of the trapping period. However, the use of a Feshbach resonance [15] would provide an effective method for suddenly reducing the meanfield interaction.

In conclusion, we have experimentally analyzed the phonon wavefunction in a Bose-Einstein condensate. Following recent theoretical work [5], the two-component character of Bogoliubov quasi-particles was observed in the frequency domain (Fig. 2). In addition, the momentum components of the phonon wavefunction were discriminated by their final momenta after the probe pulse (Fig. 1). By combining momentum and frequency selectivity, we were able to directly "photograph" the Bogoliubov transformation (Fig. 1d), demonstrating the power of Bragg spectroscopy to analyze non-trivial wavefunctions. This method may be also applicable to studying the many-body and vortex states [8] of dilute atomic Bose-Einstein condensates.

This work was funded by ONR, NSF, ARO, NASA, and the David and Lucile Packard Foundation. We are grateful to A. Brunello and S. Stringari for insightful discussions.
[1] N. N. Bogoliubov, J. Phys. (USSR) 11, 23 (1947).

[2] A. Einstein, Sitzungsber. Preuss. Akad. Wiss. Bericht 3, 18 (1925).

[3] L. D. Landau, J. Phys. (USSR) 5, 71 (1941).

[4] N. Birell and P. Davies, Quantum Fields in Curved Space (Cambridge University Press, 1982).

[5] A. Brunello, F. Dalfovo, L. Pitaevskii, and S. Stringari, Phys. Rev. Lett. 85, 4422 (2000).

[6] D. M. Stamper-Kurn, A. P. Chikkatur, A. Görlitz, S. Inouye, S. Gupta, D. E. Pritchard, and W. Ketterle, Phys. Rev. Lett. 83, 2876 (1999).

[7] J. Stenger, S. Inouye, A. P. Chikkatur, D. M. StamperKurn, D. E. Pritchard, and W. Ketterle, Phys. Rev. Lett. 82, 4569 (1999).

[8] F. Zambelli, L. Pitaevskii, D. M. Stamper-Kurn, and S. Stringari, Phys. Rev. A 61, 063608 (2000).

[9] P. B. Blakie, R. J. Ballagh, and C. W. Gardiner, preprint cond-mat/0108480.
[10] K. Huang, Statistical Mechanics (Wiley, New York, 1987).

[11] R. Onofrio, D. S. Durfee, C. Raman, M. Köhl, C. E. Kuklewicz, and W. Ketterle, Phys. Rev. Lett. 84, 810 (2000).

[12] A. P. Chikkatur, A. Görlitz, D. M. Stamper-Kurn, S. Inouye, S. Gupta, and W. Ketterle, Phys. Rev. Lett. 85, 483 (2000).

[13] S. Inouye, A. P. Chikkatur, D. M. Stamper-Kurn, J. Stenger, D. E. Pritchard, and W. Ketterle, Science 285, 571 (1999).

[14] E. W. Hagley, L. Deng, M. Kozuma, J. Wen, K. Helmerson, S. L. Rolston, and W. D. Phillips, Science 283, 1706 (1999).

[15] S. Inouye, M. R. Andrews, J. Stenger, H.-J. Miesner, D. M. Stamper-Kurn, and W. Ketterle, Nature 392, 151 (1998). 